\title{
GNSS remote sensing
}

\author{
Kegen Yu ${ }^{1 *}$, Chris Rizos ${ }^{2}$, Derek Burrage ${ }^{3}$, Andrew G Dempster ${ }^{4}$, Kefei Zhang ${ }^{5}$ and Markus Markgraf ${ }^{6}$
}

\section{Editorial}

Global Navigation Satellite System (GNSS) remote sensing comprises GNSS reflectometry (GNSS-R) and GNSS refractometry (also known as GNSS radio occultation or GNSS-RO). GNSS-R involves analyzing measurements of GNSS signals reflected from the Earth's surface, while GNSS-RO utilizes measurements of GNSS signals refracted by the atmosphere, when the slanted propagation path is close to the Earth's limb. Investigations of GNSS remote sensing of the Earth's surface and atmosphere started more than two decades ago. Many ground-based, aircraft-based, and satellite-borne experiments have been conducted, and a huge amount of experimental data has been recorded using dedicated receivers. Various methods and techniques have been proposed to process the recorded data online or offline in order to retrieve the geophysical parameters of interest. It has been proven that GNSS-RO significantly improves weather forecasting, and it has become a key component of the operational observing system. On the other hand, GNSS-R is still an experimental technique, although significant efforts are being dedicated to advance this technology, especially through a number of missions funded or to be funded by space agencies. This special issue publishes several innovative ideas, methods, and applications in GNSS remote sensing, especially in GNSS-R. The issue comprises six research papers of both theoretical studies and practice and one review article. The topics investigated include sea level measurement, snow depth estimation, snow water equivalent estimation, soil moisture estimation, buried object detection, and simplified GNSS signal processing techniques.

The review paper [1] states that the global availability of GNSS signals provides the ideal means of observing different parameters of the Earth's atmosphere, land, and ocean surface. The paper summarizes developments in theory and signal processing methodologies and mentions a number of satellite-borne, airborne, and ground-based experiments relating to GNSS reflectometry and refractometry. GNSS remote sensing is rapidly developing into a valuable

\footnotetext{
* Correspondence: kgyu@sgg.whu.edu.cn

${ }^{1}$ School of Geodesy and Geomatics, Wuhan University, Wuhan 430079, China Full list of author information is available at the end of the article
}

passive remote sensing technology, providing information on atmospheric water vapor, ocean roughness, surface wind, soil moisture, forest cover, sea ice, and snow cover. A number of space agencies - NASA, NOAA, EUMETSAT, and ESA - have already funded or will fund in the future, projects/missions which focus on a variety of GNSS remote sensing applications. This paper therefore is timely, providing an overview of the state of the art of this relatively new and, in some respects, under-utilized remote sensing technique. Also addressed are challenging issues associated with GNSS remote sensing services similar to the very successful International GNSS Service (IGS) and the performance enhancement of GNSS remote sensing to accurately and reliably retrieve a range of geophysical parameters.

Sea surface altimetry is important since mean surface level measurements provide useful information about global warming and cooling. Sea level measurement is also a direct way to detect a tsunami and other damaging ocean waves. Lofgren and Haas [2] made use of a true GNSS tide gauge, installed at the Onsala Space Observatory in Sweden. Both Global Positioning System (GPS) and Globalnaya Navigatsionnaya Sputnikovaya Sistema (GLONASS) signals were recorded during an experimental campaign of 1 month. Both the signal-tonoise ratio (SNR) analysis and the phase delay analysis were evaluated using dual-frequency (L1 and L2) GPS and GLONASS signals. The GNSS-derived sea level results were compared to independent sea level observations from a co-located pressure tide gauge. When sea surface wind speed is small and the surface is calm, the phase delay results show a better agreement with the tide gauge sea level than the SNR results. On the other hand, the SNR analysis performs better in rough sea surface conditions than the phase delay analysis. The SNR analysis is possible even during the highest wind speed observed during this campaign $(17.5 \mathrm{~m} / \mathrm{s})$, while the phase delay analysis becomes difficult for wind speeds above $6 \mathrm{~m} / \mathrm{s}$. It was also observed that for the phase delay analysis, the L1 and L2 frequencies have similar performance, whereas for the SNR analysis, the L1 band produces better performance than the L2 band which 
has a lower SNR. The results also demonstrate that GPS and GLONASS tide gauges have similar performance.

Snow water equivalent (SWE) is important for the management of water supply and flood control systems in seasonal snow-covered regions. SWE is the amount of water stored in snowpack, which is equal to the product of snow depth and density. Jacobson [3] investigated snow depth and density and hence SWE estimation using a nonlinear least squares fitting algorithm. This method utilizes the GPS interferometric reflectometry technique, and the reflection surface considered is a slightly tilted surface. A 1-day experimental campaign was conducted in a snow-covered prairie grass field, and signals associated with two GPS satellites were processed to estimate snow depth, density, and SWE. Similar to several other GNSS-R applications, the range of GPS satellite elevation angle of interest is chosen to be between $5^{\circ}$ and $30^{\circ}$, a range over which the multipath signal is strong. The experimental results indicate that the GNSS-R technique underestimates the snow depth provided by in situ measurements, while the GNSS-R-based snow density estimation produces slightly better results, which are basically within the in situ calculation range. It is intended to conduct further investigations to improve snow density, and especially snow depth, estimation performance.

Snow is important to the ecological system, climate system, and agriculture. Prior research has shown that the currently deployed geodetic GPS stations can be used to measure snow depth in an area around the antenna installation using the technique of GPS interferometric reflectometry. The advantages of such a technique include large spatial coverage and high temporal measurement sampling rate, whereas the drawback is that the geodetic equipment is expensive and the geodetic antenna is designed to significantly mitigate the multipath signal which, however, contains the desired environmental information. Through evaluating the use of GNSS interferometric reflectometry for snow depth estimation, Chen et al. [4] observed that the horizontally polarized electromagnetic wave is the desired signal for this application. A customized dipole antenna, which was horizontally polarized by properly adjusting its orientation, was built and evaluated in an experimental campaign carried out at Table Mountain, Boulder, Colorado, USA in early February 2012. In this experiment, a universal software radio peripheral was used to collect the raw data which was then processed by a software-defined receiver (SDR) to extract the L2C signal. The results demonstrate that this dipole antenna/SDR implementation scheme outperforms the geodetic GPS station-based approach.

Prior experiments using geodetic instruments equipped with circularly polarized antennas and measuring either GPS normalized reflected power or interference between the direct and reflected signals have shown a complex response to soil moisture variations. In contrast, Yan et al. [5] investigated the feasibility of estimating soil moisture from GPS L1 interference signals obtained from a low-cost commercial receiver. To maximize the interference effect, a horizontally polarized antenna receiving both direct and reflected signals with similar gain was employed. Spectral analysis of the interference signals was used to study variations in SNR frequency and phase occurring before and after rainfall. An experiment to acquire SNR data from two antennas at different heights above a flat grassy field was performed at Gardiner's Creek, Melbourne, Australia. The data were filtered and sorted by sine of the elevation angle. Spectral analysis was then used to determine the period of SNR variation. After band-pass filtering to reduce noise, the phase of the interference signal was determined, and relationships among signal frequency, phase, and soil moisture were investigated. A correlation was found between the frequency and phase estimates obtained before and after rainfall and in situ measurements of volumetric soil moisture. Additional experiments with data from continuously operated $\mathrm{PBO}$ and CORS network stations also demonstrated significant phase delays in response to rainfall and increased soil moisture. Some inconsistent results due to possible L1 signal instabilities are under investigation.

Typically, GNSS-R relies on the analysis of the reflected signal to determine the characteristics of the reflection surface and to derive the geophysical parameters. Notarpietro et al. [6] considered a new application of GNSS-R, namely the detection of metallic objects buried underground. For passive remote sensing using $\mathrm{L}$ band signals, the penetration depth varies basically from $10 \mathrm{~cm}$ to $1 \mathrm{~m}$ depending on whether the soil is wet or dry. That is, the reflected signal may contain components reflected by a shallow buried object. The authors reported a very light and low-cost GNSS receiver prototype based on an SDR approach. This receiver can be used as a payload on board small drones or unmanned aerial systems to detect metallic objects (mines or other explosive devices). Two ground-based experimental campaigns were conducted, one over sandy terrain in July 2013 and the other over grass terrain in August 2013. A round metallic plate of diameter $28 \mathrm{~cm}$ was used as the buried object, similar to the dimensions of mines and some explosives. Different scenarios including a static and a simulated moving receiver platform were tested. Significant SNR variations were observed in the presence of the buried metallic plate.

The last paper [7] is not related to a specific application of GNSS remote sensing but is about the processing of GNSS signals. A new method for acquiring GNSS signals is introduced that reduces the search in the frequency (Doppler) domain. By exploiting an energy grid search, the authors show that, particularly for strong signals, the most likely Doppler frequency can be detected prior to 
processing. By identifying the most likely candidates, the frequency search time can be significantly reduced. Reduced computational complexity is particularly desirable when it is required to process large datasets promptly to retrieve geophysical parameters in real time.

The six research papers published in this special issue represent a range of significant application areas of GNSS reflectometry, and the review paper provides a comprehensive review of the state of the art in GNSS remote sensing (both GNSS-R and GNSS-RO). We hope the interested readers will benefit from this special issue either to understand some fundamental concepts or to gain insight into some advanced techniques and challenging theoretical and practical issues, making further advances for this relatively new remote sensing technology. We would like to thank the Editor-in-Chief, Prof. Geert Leus, for enabling this special issue of EURASIP Journal on Advances in Signal Processing. The continuous support of the editorial staff of Springer is appreciated. We also thank the authors for their high-quality contributions to this special issue and to the reviewers for their valuable comments and suggestions. Researchers, especially young researchers, are encouraged to participate in the development of the fundamental theory or in the practical application of this exciting and important remote sensing area.

\footnotetext{
Author details

${ }^{1}$ School of Geodesy and Geomatics, Wuhan University, Wuhan 430079, China.

${ }^{2}$ School of Civil and Environmental Engineering, UNSW, Sydney, NSW 2052,

Australia. ${ }^{3}$ US Naval Research Laboratory, Washington, DC 20375, USA.

${ }^{4}$ School of Electrical Engineering and Telecommunications, UNSW, Sydney,

NSW 2052, Australia. ${ }^{5}$ SPACE Research Centre, RMIT University, Melbourne,

VIC 3001, Australia. ${ }^{6}$ Section Space Flight Technology, German Space

Operations Center (DLR), 82234 Wessling, Germany.
}

Received: 14 October 2014 Accepted: 14 October 2014

Published: 25 October 2014

\section{References}

1. K Yu, C Rizos, D Burrage, AG Dempster, K Zhang, M Markgraf, An overview of GNSS remote sensing. EURASIP J Adv Signal Process 2014, 134 (2014)

2. JS Lofgren, R Haas, Sea level measurements using multi-frequency GPS and GLONASS observations. EURASIP J. Adv. Signal Process. 2014, 50 (2014)

3. MD Jacobson, Estimating snow water equivalent for a slightly tilted snow-covered prairie grass field by GPS interferometric reflectometry. EURASIP J. Adv. Signal Process. 2014, 61 (2014)

4. Q Chen, D Won, DM Akos, Snow depth sensing using the GPS L2C signal with a dipole antenna. EURASIP J. Adv. Signal Process. 2014, 106 (2014)

5. $\mathrm{S}$ Yan, Z Li, K Yu, K Zhang, GPS-R L1 interference signal processing for soil moisture estimation: an experimental study. EURASIP J. Adv. Signal Process. 2014, 107 (2014)

6. R Notarpietro, SD Mattia, M Campanella, Y Pei, P Savi, Detection of buried objects using reflected GNSS signals. EURASIP J. Adv. Signal Process. 2014, 132 (2014)

7. B Soltanian, A Demirtas, ASH Ghadam, M Renfors, Reduced-complexity FFT-based method for Doppler estimation in GNSS receivers. EURASIP J. Adv. Signal Process. 2014, 143 (2014)

doi:10.1186/1687-6180-2014-158

Cite this article as: Yu et al: GNSS remote sensing. EURASIP Journal on Advances in Signal Processing 2014 2014:158.

\section{Submit your manuscript to a SpringerOpen ${ }^{\circ}$ journal and benefit from:}

- Convenient online submission

- Rigorous peer review

- Immediate publication on acceptance

- Open access: articles freely available online

- High visibility within the field

- Retaining the copyright to your article

Submit your next manuscript at $>$ springeropen.com 